\title{
WHAT WEIGHT SHOULD WEIGHTS HAVE IN INDIVIDUAL DIFFERENCES SCALING?
}

\author{
INGWER BORG \\ Rheinisch-Westfälische Technische Hochschule, Aachen, West Germany \\ and \\ JAMES C. LINGOES \\ The University of Michigan, Ann Arbor, U.S.A.
}

Recently, individual difference scaling has become one of the most active fields of research in psychometrics. Numerous models and algorithms have been proposed but relatively little evidence as to the validity of the produced representations is available so far. To shed some new light on this issue we will reanalyze some data collected by Green and Rao (1972) via PINDIS (Procrustrean INdividual DIfference Scaling). PINDIS is intimately related to the model underlying all presently available individual difference scaling algorithms but differs in important aspects which will allow a deeper insight into validity and interpretability of individual weights generated by them. To make our points clear, we will compare our results with those produced by INDSCAL which is (a) presently the most popular procedure, and (b) also the method of analysis chosen originally by Green and Rao (1972).

\section{The INDSCAL procedure}

INDSCAL has been extensively described elsewhere (Carroll and Chang, 1970; Carroll and Wish, 1974a, 1974b) and thus it seems sufficient to outline only its main characteristics here.

The problem in INDSCAL is to scale simultaneously a set of $N$ individual proximity data matrices such that their relationship can be optimally explained by the weighted Euclidean distance model, i.e., by

$$
d_{p q}^{(i)}=\left[\sum_{a=1}^{m} c_{a}^{(i)}\left(g_{p a}-g_{q a}\right)^{2}\right]^{1 / 2}
$$


where $d_{p q}^{(i)}$ is the distance between stimuli $p$ and $q$ in individual $i$ 's "private perceptual space", $g_{p a}$ is the coordinate value of $p$ on dimension $a$ in the "group space", and $c_{a}^{(i)}$ is the squared weight associated with dimension $a$. Thus, it is attempted to estimate or explain each individual's perceptual space by an appropriately weighted group space which represents some average perception over all individuals. For computational reasons, eqn. (1) is not used directly but converted to its scalar product form:

$$
b_{p q}^{(i)}=\sum_{a} g_{p a} c_{a}^{(i)} g_{q a},
$$

where $b_{p q}^{(i)}$ is the scalar product of stimuli $p$ and $q$ for individual $i$. In practice, $b_{p q}^{(i)}$ has to be estimated from the individual's proximity matrix by using Torgerson's additive constant procedure (Torgerson, 1958), since the $d_{p q}^{(i)}$ 's in eqn. (1) are only determined with respect to an arbitrary origin (if one assumes that the data are interval-scaled). We can rewrite eqn. (2) in simplifying matrix notation as

$$
B_{i}=G C_{i} G^{\prime} \text {, }
$$

where $G$ is the $n \times m$ coordinate matrix of the group space, $C_{i}$ an $m \times$ $m$ diagonal matrix of dimension weights. Note that $G$ is the same for all individuals which is indicated by the absence of the $i$-subscript.

The scaling algorithm solves eqn. (3) for the unknowns $G$ and all $C_{i}$ 's $(i=1, \ldots, N)$ such that the scalar product matrices, the $B_{i}$ 's $(i=1, \ldots$, $N$ ), are optimally explained in a least-squares sense. $G$ is normed such that its coordinate vectors have unit length. Individual differences are represented by the $C_{i}$ 's which are often conceived of as points in an $m$-dimensional space and therefore collectively called "subject space" $(S S)$. An individual's communality between his private perceptual space (i.e., in INDSCAL: his $B_{i}$ ) and the scalar products in his reconstructed or estimated space is represented by the squared length of his weight vector in $S S$. Although this is strictly true if and only if the coordinate vectors in $G$ are orthogonal, this is usually a good approximation.

The model in eqn. (3) has been generalized by dropping the diagonality constraint for $C_{i}$ and merely requiring that it be positive (semi-)definite. The resulting $C_{i}$ can then be further decomposed into

$$
C_{i}=T_{i} \Omega_{i} T_{i}^{\prime},
$$

where $T_{i}$ is orthogonal and $\Omega_{i}$ diagonal. This is Carroll and Chang's (1972) IDIOSCAL model which can be conceived of geometrically as an orthogonal, "idiosyncratic" rotation of $G$ by $T_{i}$, followed by a dimensional weighting by $\Omega_{i}$. Obviously, this factoring of $C_{i}$ is not 
unique and, not surprisingly, other decompositions have also been proposed (Harshman, 1972; Tucker, 1972).

\section{Problems associated with the Green-Rao approach}

The described approach to the interpretation of the subject space is standard in the sense that it is assumed that the scatter of points represents meaningful or useful information about individual differences (cf., e.g., Carroll and Wish, 1974a, b; Wish and Carroll, 1974). There are, however, two major problems associated with such an assumption and analysis:

(1) The first problem is a more technical one related to the meaning of the distances among points in SS. In an INDSCAL-SS - defined by Carroll and Chang (1970) as the configuration of the $c_{\mathbf{a}}^{(i)}$ 's $(a=1, \ldots, m)$ for all $i$ 's $(i=1, \ldots, N)$ - the distances among the points represent approximately "profile distances" between reconstructed scalar products from the respective private perceptual spaces (Carroll and Wish, 1974a), i.e., between the elements of $\hat{B}_{i}$ and $\hat{B}_{j}$ :

$$
\sum_{a}\left(c_{a}^{(i)}-c_{a}^{(j)}\right)^{2} \approx \sum_{p>q}\left(\hat{b}_{p q}^{(i)}-\hat{b}_{p q}^{(j)}\right)^{2},
$$

where $i$ and $j$ indicate two different individuals. Although this is usually a very good approximation, it is nevertheless not perfect and, in particular, not easily interpretable. However, there exists a psychologically more attractive and also mathematically exact equivalence relation between distances based on the square roots of the weights and the interconfigurational distance $d\left(\hat{B}_{i}, \hat{B}_{j}\right)=d\left(G C_{i}^{1 / 2}, G C_{j}^{1 / 2}\right)$ :

$$
\sum_{a}\left(\sqrt{c_{a}^{(i)}}-\sqrt{c}_{a}^{(j)}\right)^{2}=\sum_{a} \sum_{p}\left(g_{p a} \sqrt{c}_{a}^{(i)}-g_{p a} \sqrt{c_{a}^{(j)}}\right)^{2} .
$$

In addition, the cosine between vectors in this transformed space represents the correlation between the estimated private perceptual spaces of $i$ and $j$. Although the $S S$ will thus have simpler properties if it is normed appropriately, a more fundamental problem remains unresolvable: the squared length of each subject vector represents the communality of a $B_{i}$ and its estimate $\hat{B}_{i}=G C_{i} G^{\prime}$ if and only if $G$ is column-wise orthogonal, i.e., uncorrelated. Related to this point is the fact that the square of a component of each weight vector represents the communality contribution of the respective dimension if and only if $G$ is column-wise orthogonal (Lingoes et al. 1977). This is important since one might prefer to determine the similarity among individuals on the basis of their 
configurational communality, that is their common explained variance, rather than on the basis of inter-configurational distances. If the coordinate vectors of $G$ are correlated then the distances computed on the squared SS will not only represent these communality differences but also some interaction effects whose psychological meaning remains totally obscure.

In the 3-dimensional $G$ reported by Green and Rao (1972), the coordinate axes are not orthogonal: correlations are $r_{12}=.043, r_{13}=-.440$, and $r_{23}=.330$, where $r_{a b}$ indicates a correlation coefficient between axes $a$ and $b$. Thus, if a cluster analysis were run on the associated squared $S S$, it would use distances which do not only represent intersubject similarity but also the non-orthogonality of $G$. This is clearly not a satisfactory state of affairs. Even for small correlations this may produce misleading clusters since the algorithms are usually very sensitive to even small displacements of points if the space is not clearly discontinuous.

(2) The second problem involved in the Green-Rao approach is less obvious: it is related to the validity of the point scatter in $S S$. The meaningful information in the data consisted of comparative disiances and, consequently, it seems natural to first ask what proportion of this information could be explained by transformations which preserve the relative distances before distorting weights are used. The validity and interpretability of the dimension weights must be evaluated against the amount of variance explained by admissible transformations. Clearly, if no substantial fit improvement can be achieved by differential weights, then one should rather assume unit weights, or, in other words, one should interpret inter-individual differences in the $S S$ to be more a consequence of the mathematical procedures than of reliable inter-subject differences. It will be shown below that the Green-Rao data lead to a considerable scatter of points in $S S$, but that the additional explanation that is achieved by these differential weights is negligible, and thus the $S S$ is insignificant and misleading if looked upon without knowledge of the performance of admissible transformations. In INDSCAL, this information is not available.

\section{The PINDIS model}

The PINDIS model and algorithm have been described elsewhere in considerable detail (Lingoes et al. 1977; Lingoes and Borg, 1976b) and it suffices therefore to restate here only its relevant features.

In PINDIS, it is necessary first to scale the individual proximity ma- 
trices by some multidimensional procedure which yields an $n \times m_{i}$ configuration $X_{i}$ for each subject $i(i=1, \ldots, N)$, where $n$ indicates the number of points, $m_{i}$ the dimensionality of the solution for individual $i$. These $X_{i}$ 's are then used as input to PINDIS. In PINDIS, each $X_{i}$ is centered at the origin, and the total configuration is scaled to unit length, transformations which preserve relative distances. An $n \times \max \left(m_{i}\right)$ centroid configuration $Z$ which represents the perceptual space of the average subject is then computed by a method similar to the one proposed by Gower (1975). The norm of $Z$ is the communality of all individuals. Each $X_{i}$ is subsequently rotated/reflected such that the sum of squared distances between corresponding points in $Z$ and $X_{i}$ is a minimum. These transformations are admissible in the sense that they preserve all inter-point distances. Finally, a measure of configurational similarity, Lingoes and Schönemann's (1974) $S$, which reflects the amount of variance of each $X_{i}$ (fitted to $Z$ by such an orthogonal Procrustean rotation) that can be explained by $Z$, is computed. $S$ is related to a correlation coefficient as $S^{2}=1-r^{2}\left(Z, X_{i}\right)$ and, thus, is not affected by differences in the overall scale of $Z$ and $X_{i}$. Expressed differently: $S$ implicitly takes a central dilation/contraction factor on either $Z$ or $X_{i}$ into account which is also an admissible transformation since only the relations of the distances in $X_{i}$ are considered to be meaningful.

PINDIS then finds weights $w_{a}^{(i)}$ for the dimensions of $Z$ for each individual which maximize the correspondence between optimally reoriented $X_{i}$ 's and the individually weighted $Z$ 's, the $Z W_{i}$ 's, where $W_{i}$ is an $m \times m$ diagonal matrix with elements $w_{a}^{(i)}(a=1, \ldots, m)$. This step is analogous to the INDSCAL approach except that the minimization is in terms of distances between configurations and not scalar products derived from them. It may also be noted that $Z$ was previously optimally rotated and that this position - as is G's position in INDSCAL is unique up to axes permutations. Obviously such a dimension-weighting transformation is not isotonic anymore, i.e., distance relations are now, in general, changed. We gain some information about the explanatory power of this weighting over and above the isotonic approach used previously by the increment in the attained fit between $X_{i}$ and $Z W_{i}$ : if the communality is not substantially better than previously, then the weights are clearly not likely to represent valid increments in psychological information. Nevertheless, in spite of little overall improvement of $S$, some individuals may be definitely better representable by a weighted $Z$. In addition, PINDIS calculates individually optimal $Z$ 's, i.e., reoriented (rotated) $Z$ 's for each individual $\left(Z_{i}\right)$, which may also be substantially more powerful predictors for some or all subjects than the average-subject $Z$. Thus, not only can the overall importance of the 
weighting - and, consequently, of the $S S$ - be evaluated but important inferences about the group's homogeneity under this transformation can also be attained. At the third stage of a PINDIS analysis, each point in $X_{i}$ and $Z$ is conceived of as the terminus of a vector emanating from the origin. After an optimal reorientation of $X_{i}$, each vector in $Z$ is then weighted by $v_{p}^{(i)}(p=1, \ldots, n)$ such that the squared distance between its endpoint and the one of the corresponding vector in $X_{i}$ is a minimum. Again, a fit value is computed which indicates the performance of this approach, i.e., the attained fit between $X_{i}$ and $V_{i} Z$, where $V_{i}$ is diagonal with $v_{p}^{(i)}(p=1, \ldots, n)$ as its elements. If considerable fit improvement has been achieved by $V_{i} Z$ over $Z W_{i}$ and/or the isotonic fitting, it is likely that this transformation represents some real interindividual differences. The vector-weighting approach is particularly suited to detect cascs in which the $X_{i}$ 's are "scrambled" with respect to the average subject's configuration $(Z)$ and thus enables a deeper understanding of the group's homogeneity and the representativity of $Z$ for all individuals in the study. This will become more evident below.

The fourth step of PINDIS combines both previous transformations into one composite approach, finding $V_{i}$ and $W_{i}$ simultaneously. $Z$ is thus mapped into $V_{i} Z W_{i}$. Since this last analysis is not of much use here, it will not be further discussed.

\section{Reanalysis of the Green-Rao data [2]}

As in the Green and Rao's original analysis, the data were initially processed by TRICON in order to generate unconditional proximity matrices. Since we used a more recent version of TRICON, however, the results of this step differ slightly from those reported by Green and Rao (1972). In particular, the newer program eliminated one subject (Nr. 17-1 in the G-R notation) altogether because of incomplete data. We have, therefore, only 41 subjects. (The slight differences in the proximity data are, of course, quite irrelevant for the purpose of this article.)

For a PINDIS analysis, the $4115 \times 15$ data matrices where then scaled via SSA-I (Guttman, 1968; Lingoes, 1973) in 2-dimensional spaces. The resulting configurations are the $X_{i}$ 's. The stress coefficient was .1324 on the average with a standard deviation of .0382 . Although this is not a particularly good representation, it is sufficient for the present purpose.

A PINDIS analysis of the $X_{i}$ 's generates first the centroid configuration $Z$ (Fig. 1) which corresponds well to the group space reported by 


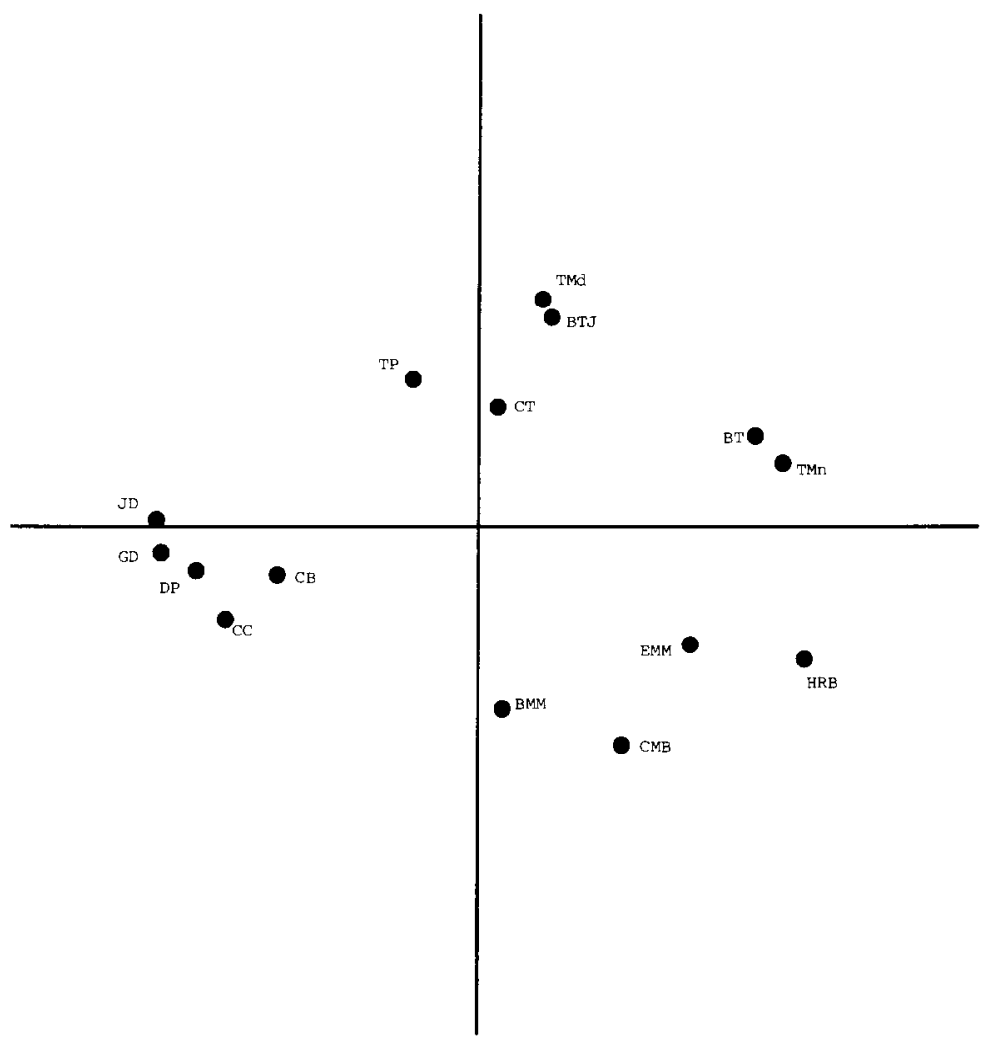

Fig. 1. Centroid configurations $(Z)$ from PINDIS analysis.

Green and Rao (1972). (For a list of the items used in the Green-Rao study, see Table I). Fitting the $X_{i}$ 's to this $Z$ yields an average communality of $r^{2}\left(X_{i}, Z\right)=.7196$. This means that some $72 \%$ of the variance in all individual configurations can be explained by $\mathrm{Z}$ without any distortions of the relative distances among the points. In order to be able to compare these results to those attainable from INDSCAL, Euclidean distances for all $X_{i}$ 's were computed and analyzed via INDSCAL. The produced group space $G$ is represented in Fig. 2. The correlation of $G$ 's coordinate vectors is $r=.3056$. As is obvious from visual inspection alone, $Z$ and $G$ differ only in their orientation and in their norming. A more objective analysis via MFIT (Schönemann and Carroll, 1970; Lingoes, 1973) validates this impression: a clockwise rotation of $Z$ by $26.7^{\circ}$ and a central dilation by 1.39 transforms $Z$ such that the fit between the two spaces is $r^{2}=.9697$. However, $G$ and $Z$ differ also in their norming: in $G$, each coordinate vector has length one, whereas $Z$ represents directly the perceptual space of the average subject. Reorienting $Z$ optimally by rotating it by $-3.38^{\circ}$ and weighting $G$ 's axes by 


\section{TABLE I}

Food items used in Green Rao study

\begin{tabular}{rll}
\hline No. & Food item & Code \\
\hline 1 & Toast pop-up & TP \\
2 & Buttered toast & BT \\
3 & English miffin and marganine & FMM \\
4 & Jelly donut & JD \\
5 & Cinnamon toast & CT \\
6 & Blueberry muffin and margarine & BMM \\
7 & Hard rolls and butter & HRB \\
8 & Toast and marmalade & TMd \\
9 & Buttered toast and jelly & BTJ \\
10 & Toast and margarine & TMn \\
11 & Cinnamon bun & CB \\
12 & Danish pastry & DP \\
13 & Glazed donut & GD \\
14 & Coffee cake & CC \\
15 & Corn muffin and butter & CMB \\
\hline
\end{tabular}

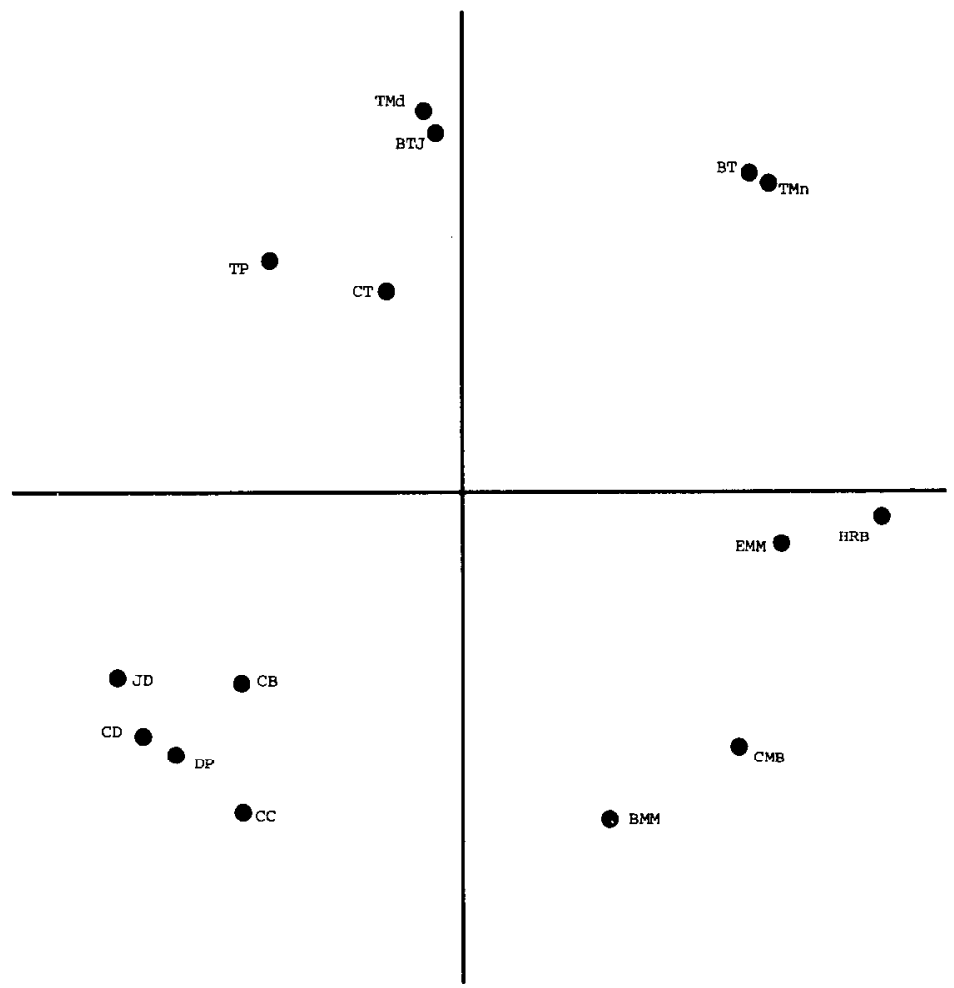

Fig. 2. Group space $(G)$ from INDSCAL analysis. 
1.13 and .808 yields a fit of $r^{2}=.9968$ between these two configurations. Since $G$ and $Z$ are both uniquely oriented in the sense of having an optimal position with respect to the dimension-weighting transformation, their differences are related to differences in norming and in the optimization criteria, a direct function of the coordinates in PINDIS, a derived function, i.e., scalar products, in INDSCAL. (It should be noted, however that using Euclidean distances in the $X_{i}$ 's as input for INDSCAL eliminates the problems associated with its scalarproduct eștimation procedure.)

As a next step, dimension weights are calculated for optimally reoriented $X_{i}$ 's. This leads to an average fit of $r^{2}\left(X_{i}, Z W_{i}\right)=.7456$. The improvement over the fit attainable by strictly admissible transformations is only $2.6 \%$. The resulting $S S$ (Fig. 3 ) is, therefore, not likely to represent any real differential information. The scatter of subject points about the origin should not be interpreted substantively, in particular, since the introduction of two free parameters (the dimension weights) should, in general, lead to some fit improvement anyway.

The corresponding $S S$ for INDSCAL (Fig. 4) shows no less variability and might be quite misleading since in INDSCAL nothing is known about the performance of admissible transformations. This may then apart from the technical issues discussed above - account for the difficulties that Green and Rao met in interpreting the clusters derived from the INDSCAL-SS.

If the fit improvement was substantial, on the other hand, one could
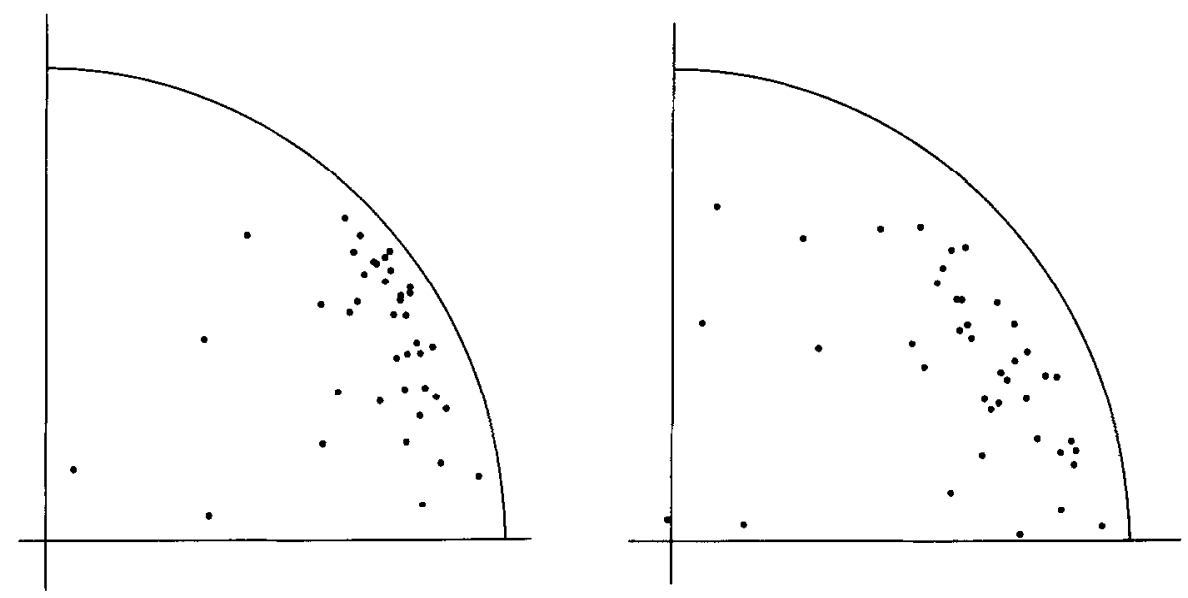

Fig. 3. Subject space (SS) associated with $Z$ (PINDIS).

Fig. 4. Subject space $(S S)$ associated with $G$ (INDSCAL). 
use a cluster analysis for a PINDIS-SS without the interpretational problems involved in INDSCAL, since the distances in a PINDIS-SS always represent the distances between all corresponding points in estimated private perceptual spaces. Furthermore, the squared length of a subject vector in PINDIS corresponds to this subject's communality independent of the orthogonality properties of $Z^{-}$. This means that the contribution of a dimension to the total communality will also be known exactly, whereas in INDSCAL this is only true if $G$ is orthogonal.

Note also that in the INDSCAL-SS (Fig. 4) there is one subject with a negative weight for one dimension. This indicates that the model is not correct for this individual. In PINDIS, negative dimension weights are entirely admissible: they simply mean that for such individuals the point-projections are reserved in some way relative to those in $Z$.

\section{Additional information provided by PINDIS}

So far, only a small fraction of the potentially useful information provided by PINDIS has been reported. Not only is the average fit value interesting but, of course, also the individual coefficients upon which it is based. In addition, we can also check if an individually reoriented $Z$, $Z_{i}$, allows a much better explanation of $X_{i}$ for some individuals. To illustrate these points, the fit values (communalities) for all individuals are presented in Table II.

For some individuals, e.g., 5, 11, 31 and 32 , one can see that some fit improvement is possible by "idiosyncratic" orientations of $Z$. (Note that these rotations are unique in contrast to those computed by IDIOSCAL.) On the average, however, $Z_{i}$ 's do not account for much more variance in the $X_{i}$ 's than $Z$. It is interesting to note that, for example, individuals 20,32 , and 38 are only very poorly explained by $Z W_{i}$. In INDSCAL, where the communalities, i.e., the squared correlations between $B_{i}$ and $G C_{i} G^{\prime}$, are $.027, .223$, and .001 for these subjects, respectively, one might conclude that these individuals use other dimensions than those in $G$. Carroll (1972) comments on this matter as follows: "A subject precisely at the origin [of the $S S$ ] . . is not accounted for at all in this analysis; he has nothing at all in common with the other subjects, or, perhaps, is simply responding randomly (p.111)." This, however, is not necessarily true: the private perceptual spaces of these subjects may merely be scrambled relative to $Z$. The vectorweighting analysis in PINDIS, the third step described above, reveals this quite clearly. For subject 20 , for example, we find that all the 
TABLE II

Communalities between individual configurations $\left(X_{i}\right.$ 's) and $Z$ for various transformations in PINDIS

\begin{tabular}{|c|c|c|c|c|}
\hline Subj. & $\left(X_{i}, Z\right)$ & $\left(X_{i}, Z W_{i}\right)$ & $\left(X_{i}, Z_{i} W_{i}\right)$ & $\left(X_{i}, V_{i} Z\right)$ \\
\hline 1 & .7999 & .8005 & .8008 & .8335 \\
\hline 2 & .8520 & .8713 & .8714 & .8926 \\
\hline 3 & .9088 & .9112 & .9159 & .9519 \\
\hline 4 & .9194 & .9222 & .9283 & .9488 \\
\hline 5 & .5669 & .6811 & .7352 & .8410 \\
\hline 6 & .8939 & .9056 & .9065 & .9376 \\
\hline 7 & .8376 & .8469 & .8480 & .8692 \\
\hline 8 & .8365 & .9014 & .9032 & .8900 \\
\hline 9 & .8518 & .8520 & .8849 & .8977 \\
\hline 10 & .7369 & .7404 & .7439 & .8407 \\
\hline 11 & .7765 & .7958 & .8803 & .8631 \\
\hline 12 & .7044 & .7188 & .7649 & .8099 \\
\hline 13 & .7833 & .9152 & .9161 & .9154 \\
\hline 14 & .7772 & .7814 & .8020 & .9219 \\
\hline 15 & .8982 & .9175 & .9175 & .9339 \\
\hline 16 & 6199 & .6698 & .6747 & .7751 \\
\hline 17 & .6871 & .7765 & .7805 & .8015 \\
\hline 18 & .7881 & .8174 & .8324 & .8821 \\
\hline 19 & .8050 & .8469 & .8493 & .8540 \\
\hline 20 & .1118 & .1307 & .1307 & .7782 \\
\hline 21 & .6179 & .6310 & .6631 & .6754 \\
\hline 22 & .9222 & .9429 & .9470 & .9588 \\
\hline 23 & .8770 & .8794 & .9005 & .9184 \\
\hline 24 & .8721 & .8777 & .8785 & .8866 \\
\hline 25 & .5101 & .5135 & .5419 & .8383 \\
\hline 26 & .6827 & .6884 & .6895 & .7731 \\
\hline 27 & .8251 & .8280 & .8385 & .8712 \\
\hline 28 & .7198 & .7268 & .7644 & .8292 \\
\hline 29 & .8493 & .8931 & .8936 & .9199 \\
\hline 30 & .8593 & .8978 & .9068 & .9289 \\
\hline 31 & .3929 & .4067 & .4695 & .8143 \\
\hline 32 & .2728 & .2994 & .3642 & .6585 \\
\hline 33 & .8498 & .8700 & .8776 & .9448 \\
\hline 34 & .7973 & .8299 & .8451 & .8860 \\
\hline 35 & .5126 & .6170 & .6623 & .6976 \\
\hline 36 & .6076 & .6212 & .6894 & .6806 \\
\hline 37 & .8137 & .8192 & .8322 & .8786 \\
\hline 38 & .0192 & .0262 & .0331 & .4690 \\
\hline 39 & .7077 & .7426 & .7478 & .8903 \\
\hline 40 & .7824 & .8004 & .8019 & .8306 \\
\hline 41 & .8559 & .8581 & .9178 & .9178 \\
\hline mean & .7196 & .7456 & .7647 & .8465 \\
\hline
\end{tabular}


weights differ considerably from +1 , among them 10 negative weights for the 15 points in $Z$. This means that in his perceptual space the food items are clustered differently. The item TMn, for example, is weighted by -1.05 which reverses the direction of its vector and positions TMn in the opposite quadrant. Thus, the item vectors are "flipped over", stretched and shrunk such that their termini belong to different item groups. The communality for subject 20 under $V_{i} Z$ is thereby increased to $r^{2}=.7782$, an improvement of $65 \%$ over $Z W_{i}$. Subject 20 could, therefore, have something in common with the other individuals, namely the dimensions, although not the clusters of $Z$.

We now demonstrate graphically the function of vector weights for a relatively simple case. Individual 14 requires three weights substantially different from +1 in order to fit $Z$ to his $X_{i}$; all other weights are sufficiently close to +1 to be ignored here in this illustration. The respective weights are 1.7 for $\mathrm{TP},-1.4$ for $\mathrm{CT}$, and 1.6 for BMM, which leads to the displacements of these points represented in Fig. 5.

The communality is increased by such weighting to .9219 from that of .7814 under the dimension-weighting transformation. As simple as this case is, it shows clearly that subject 14 associates CT with another group of items, in particular with BMM, whereas the average subject perceives CT to be most closely related to TP.

The vector-weighting transformations for individual 14 are also interesting in another respect: the items TP, CT, and BMM are actually those stimuli in $Z$ which require weights different from +1 for almost all individuals, whereas other item-points in $Z$ represent most corresponding individual points with very little shifting. Furthermore, there are no simple groups of weight patterns for these items among the individuals that would allow one to cluster subjects into types of similar perception. It seems that the consensus as to where these items belong is low. On the other hand, inter-individual agreement is quite high in the sense that CT, TP, and BMM are always displaced such that the approximate ellipse that $Z$ 's points form is preserved. Recognizing this higher-order invariance, one could, therefore, also try to interpret $Z$ as a circumplex rather than using the cluster and dimensional approach chosen by Green and Rao.

Cases like these also shed some additional light on the interpretability of $Z$ since a large number of subjects with vector weight patterns consisting of coefficients very different from +1 , in particular negative ones, indicate that $Z$ might not represent any subject's private perceptual space, but rather some confounding of essentially qualitatively different individuals. The consequences in such a case are quite clear: one should cluster individuals first into groups of relatively homogeneous 


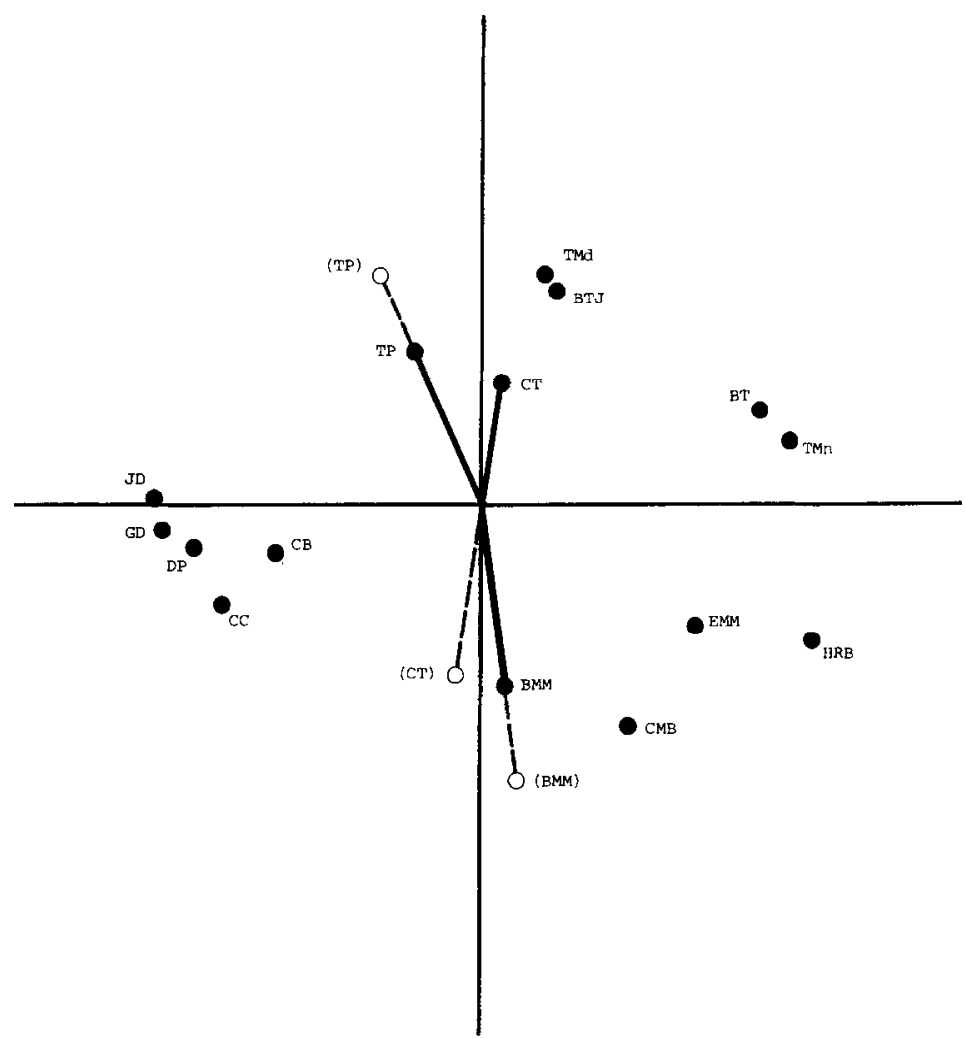

Fig. 5. Function of vector weights: solid lines represent vectors in $Z$, dashed lines vectors in $V_{i} Z$.

perceptions on the basis of their vector weights and then investigate these groups separately.

On the average, we find for the present data a communality of $r^{2}=$ .8465 for the $V_{\mathrm{i}} Z$ approach which is over $10 \%$ better than the dimension-weighting transformation. Thus, it seems likely that, in spite of the substantially higher numbers of free parameters ( 15 vs. 2 for the dimension weighting), some real structural inter-individual differences relative to $Z$ are given in the data.

\section{Discussion}

As we have already repeatedly pointed out (Borg and Lingoes, 1976; Lingoes and Borg, 1976a) we recommend using PINDIS in a confirmatory approach rather than in a purely exploratory way as implemented in this paper. In practical terms, this means that one should rotate $Z$ 
first to a position of hypothesized meaning and then determine all other transformations relative to this $Z$. This could be done by running PINDIS once, fitting the generated $Z$ Procrustean-wise to some target matrix which represents the hypothesis, and using this reoriented $Z$ then as a fixed hypothesis configuration in a second PINDIS run (PINDIS provides an option for the usage of such an externally generated $Z$.).

Although we have not addressed ourselves to substantive issues in this article, the PINDIS analysis is clearly suggestive that more could be said about the data than is possible by a dimensional or cluster approach. Some other potentially useful interpretational techniques are discussed in Lingoes et al. (1977). A study aiming at substantive findings could also profit considerably from a detailed facet-theoretical approach (cf. also Lingoes et al. 1977).

\section{Notes}

1 Only a small fraction of Green and Rao's analysis is referred to in this paper. Our intention is merely to remind the reader of some points that are relevant in this context.

2 We are grateful to Professor Green for sending us the complete data deck necessary for this study.

\section{References}

Borg, I. and Lingoes, J.C. (1976). “A direct transformational approach to multidimensional analysis of three-way data matrices: theory and appiications," Michigan Mathematical Psychology Program Technical Report MMPP-76-1: 1-33.

Carmone, F.J., Green, P.E., and Robinson, P.J. (1968). "TRICON-An IBM 360/65 Fortran IV program for the triangularization of conjoint data," Journal of Marketing Research, (1970). 5: 219-220.

Carroll, J.D., (1972). "Individual differences and multidimensional scaling," in R.N. Shepard, A.K. Romney, and S.B. Nerlove (Eds.), Multidimensional Scaling, Vol. I, pp. 105-155. New York: Seminar Press.

Carroll, J.D. and Chang, J.J. (1970). "Analysis of individual differences in multidimensional scaling via an $N$-way generalization of "Eckart-Young" decomposition," Psychometrika 35: 283 - 320.

Carroll, J.D. and Chang, J.J. (1972). "IDIOSCAL (Individual Difference In Orientation $S C A L$ ing): A generalization of INDSCAL allowing IDIOsyncratic reference systems as well as an analytic approximation to INDSCAL," Paper presented at meetings of the Psychometric Society, Princeton, N.J., March 1972.

Carroll, J.D. and Wish, M. (1974a). "Multidimensional perceptual models and measurement methods," in E.C. Carterette and M.P. Friedman (Eds.), Handbook of Perception, Vol. II, pp. 391-447. New York: Academic Press.

Carroll, J.D. and Wish, M. (1974b). "Models and methods for three-way multidi- 
mensional scaling," in D.H. Krantz, R.C. Atkinson, D.R. Luce, and P. Suppes (Eds.), Contemporary Developments in Mathematical Psychology, Vol. II, pp. 57-105. San Francisco: W.H. Freeman.

Gower, J.C. (1975). "Generalized Procrustes analysis," Psychometrika 40: 33-51.

Green, P.E. and Rao, V.R. (1972). Applied Multidimensional Scaling. Hindsdale, Ill.: Dryden Press.

Guttman, L. (1968). "A general nonmetric technique for finding the smallest coordinate space for a configuration of points," Psychometrika 33:469-506.

Harshman, R.A. (1972). "PARAFAC2: Mathematical and technical notes," U.C.L.A. Working Papers in Phonetics, 22.

Howard, N. and Harris, B. (1966). A hierarchical grouping routine, IBM 360/65 FORTRAN IV program. Report University of Pennsylvania Computer Center, October 1966.

Lingoes, J.C. (1973). The Guttman-Lingoes Nonmetric Program Series. Ann Arbor, Mi.: Mathesis Press.

Lingoes, J.C. and Borg, I. (1976a) "Optimal solutions for dimension and vector weights in PINDIS," Michigan Mathematical Psychology Program Technical Report MMPP-76-4: 1-18.

Lingoes, J.C. and Borg. I. (1976b). Procrustean Individual Difference Scaling," Journal of Marketing Research 13: 406-407.

Lingoes, J.C., Guttman, L., and Roskam, E.E. (1977). Geometric Representations of Relational Data: With Social Science Applications. Ann Arbor, Mi.: Mathesis Press.

Lingoes, J.C. and Schönemann, P.H. (1974). "Alternative measures of fit for the Schönemann-Carroll matrix fitting algorithm," Psychometrika 39:423-427.

Schönemann, P.H. and Carroll, R.M. (1970). "Fitting one matrix to another under choice of a central dilation and a rigid motion," Psychometrika 35:245-255.

Torgerson, W.S. (1958) Theory and Methods of Scaling. N.Y.: Wiley.

Tucker, L.R. (1972). "Relations between multidimensional scaling and three-mode factor analysis," Psychometrika 37:3-27.

Wish, M. and Carroll, J.D. (1974). "Applications of individual differences scaling to studies of human perception and judgment," in E.C. Carterette and M.P. Friedman (Eds.), Handbook of Perception, Vol. II, pp. 449-491. New York: Academic Press. 